\title{
EXACT PROBABILITY DISTRIBUTION FUNCTIONS OF SOME RENYI TYPE STATISTICS ${ }^{1}$
}

\section{MIKLÓS CSÖRGÖ2}

1. Summary. Let $F(x)$ be the continuous distribution function of a random variable $X$, and $F_{n}(x)$ be the empirical distribution function of a random sample $X_{1}, \cdots, X_{n}$, taken on $X$. Using the method of Birnbaum and Tingey [3] we derive exact probability distribution functions for the random variables

$$
\begin{gathered}
\sup _{F(x) \leqq D}\left\{F(x)-F_{n}(x)\right\} /\{1-F(x)\}, \\
\sup _{a \leqq P(x)}\left\{F_{n}(x)-F(x)\right\} / F(x), \\
\sup _{P_{n}(x) \leqq b}\left\{F(x)-F_{n}(x)\right\} /\left\{1-F_{n}(x)\right\} \text { and } \sup _{a \leqq P_{n}(x)}\left\{F_{n}(x)-F(x)\right\} / F_{n}(x)
\end{gathered}
$$

where $0<a<1,0<b<1$, and also for the random variables

$$
\begin{gathered}
\sup _{P(x)<1}\left\{F(x)-F_{n}(x)\right\} /\{1-F(x)\}, \\
\sup _{0<F(x)}\left\{F_{n}(x)-F(x)\right\} / F(x), \\
\sup _{P_{n}(x)<1}\left\{F(x)-F_{n}(x)\right\} /\left\{1-F_{n}(x)\right\} \text { and } \sup _{0<P_{n}(x)}\left\{F_{n}(x)-F(x)\right\} / F_{n}(x) .
\end{gathered}
$$

2. Introduction and some preliminary results. Let $Y=F(x)$. Then $Y$ is a uniformly distributed random variable on $(0,1)$ and we have $Y_{1}, \cdots, Y_{n}$ as a random sample on $Y$ resulting from this transformation. Let $Y_{1}^{*}<Y_{2}^{*}<\cdots<Y_{n}^{*}$ be the same set of random variables arranged in ascending order (called: order statistics) which determines the empirical distribution function

$$
G_{n}(Y)=\left\{\begin{array}{cl}
0 & \text { for } Y<Y_{1}^{*} \\
k / n & \text { for } Y_{k}^{*} \leqq Y<Y_{k+1}^{*}, \\
1 & \text { for } Y_{n}^{*} \leqq Y .
\end{array}\right.
$$

We are going to need the following result of Birnbaum and Tingey [3]:

Received by the editors April 28, 1964.

1 This research was partially supported by the Office of Naval Research 042-023, Contract Nonr 1858(05) to Princeton University.

2 The author is now at McGill University. 


$$
\begin{aligned}
& P\left\{\sup _{-\infty<x<+\infty}\left(F(x)-F_{n}(x)\right)<\epsilon\right\}=P\left\{\sup _{0<Y<1}\left(Y-G_{n}(Y)\right)<\epsilon\right\} \\
& =n ! \int_{Y_{0}=0}^{*} \int_{Y_{1}^{*}}^{1 / n+\epsilon} \cdots \int_{Y_{k}^{*}}^{k / n+\epsilon} \int_{Y_{k+1}^{*}}^{1} \cdots \int_{Y_{n-2}^{*}}^{1} \int_{Y_{n-1}^{*}}^{1} \\
& \cdot d Y_{n}^{*} d Y_{n-1}^{*} \cdots d Y_{k+2}^{*} d Y_{k+1}^{*} \cdots d Y_{2}^{*} d Y_{1}^{*} \\
& =1-\sum_{j=0}^{k}\left(\begin{array}{l}
n \\
j
\end{array}\right)\left(1-\epsilon-\frac{j}{n}\right)^{n-j}\left(\epsilon+\frac{j}{n}\right)^{j-1} \epsilon=P_{n}(\epsilon),
\end{aligned}
$$

where $k=[n(1-\epsilon)]=$ greatest integer contained in $n(1-\epsilon)$ and $0<\epsilon \leqq 1$.

Smirnov [6] obtained the asymptotic expression

$$
\lim _{n \rightarrow \infty} P_{n}\left(z(n)^{-1 / 2}\right)=1-e^{-2 z^{2}} \text {. }
$$

The following lemmas are going to be useful.

LEMMA 1. For all real $\alpha, \beta$ and integer $n \geqq 0$

$$
(\beta-n) \sum_{i=0}^{n}\left(\begin{array}{l}
n \\
i
\end{array}\right)(\alpha+i)^{i}(\beta-i)^{n-i-1}=(\alpha+\beta)^{n} .
$$

Relation (2.4) was proved by Abel [1, Vol. 1, p. 102]. For $b=n$ the left-hand term is defined as the limit for $b \rightarrow n$.

Lemma 2. For all real $\alpha, \beta$ and integer $n>0$

$$
\begin{aligned}
& (\alpha-1)(\beta-n) \sum_{i=0}^{n} \frac{1}{i+1}\left(\begin{array}{c}
n \\
i
\end{array}\right)(\alpha+i)^{i}(\beta-i)^{n-i-1} \\
& =\frac{1}{n+1}\left[(\alpha+\beta)^{n}(\alpha+\beta-n-1)-(\beta+1)^{n}(\beta-n)\right]
\end{aligned}
$$

Relation (2.5) was proved by Birnbaum and Pyke (Lemma 3 of [2]).

Lemma 3. For all real $\alpha \neq 1, \beta \neq n$ and integer $n>0$

$$
\sum_{i=0}^{n-1}\left(\begin{array}{c}
n \\
i+1
\end{array}\right)(\alpha+i)^{i(\beta-i)^{n-i-1}}=\frac{(\alpha+\beta)^{n}-(\beta+1)^{n}}{\alpha-1}
$$

Proof of Lemma 3. We have

$$
\left(\begin{array}{c}
n+1 \\
i+1
\end{array}\right)-\left(\begin{array}{c}
n \\
i
\end{array}\right)=\left(\begin{array}{c}
n \\
i+1
\end{array}\right)
$$


and thus the left-hand side of (2.6) can be written as

$$
\begin{aligned}
\sum_{i=0}^{n} & \left\{\left(\begin{array}{l}
n+1 \\
i+1
\end{array}\right)-\left(\begin{array}{c}
n \\
i
\end{array}\right)\right\}(\alpha+i)^{i}(\beta-i)^{n-i-1} \\
& =(n+1) \sum_{i=0}^{n} \frac{1}{i+1}\left(\begin{array}{c}
n \\
i
\end{array}\right)(\alpha+i)^{i}(\beta-i)^{n-i-1} \\
& -\sum_{i=0}^{n}\left(\begin{array}{l}
n \\
i
\end{array}\right)(\alpha+i)^{i}(\beta-i)^{n-i-1}
\end{aligned}
$$

We can directly apply now Lemma 2 and Lemma 1 and statement of Lemma 3 follows immediately.

3. Exact probability distributions of random variables of $\$ 1$. TheOREM 1.

$$
P\left\{\sup _{r(x) \leqq 0} \frac{F(x)-F_{n}(x)}{1-F(x)}<\epsilon\right\}=N(\epsilon, b, n)
$$

where

$$
\begin{aligned}
N(\epsilon, b, n) & =1-\sum_{j=0}^{k} K(\epsilon, j, n) \\
& =\sum_{j=k+1}^{n} K(\epsilon, j, n),
\end{aligned}
$$

$\epsilon>0$ and $b$ is such that $0<\epsilon /(1+\epsilon) \leqq b$, and where $k=[n\{(1+\epsilon) b-\epsilon\}]$, and $K(\epsilon, j, n)$ is defined as

$K(\epsilon, j, n)$

$$
=\left(\begin{array}{l}
n \\
j
\end{array}\right)\left(1-\frac{j}{n(1+\epsilon)}-\frac{\epsilon}{1+\epsilon}\right)^{n-j}\left(\frac{j}{n(1+\epsilon)}+\frac{\epsilon}{1+\epsilon}\right)^{j-1} \frac{\epsilon}{1+\epsilon}
$$

and for $j=n, K(\epsilon, j, n)$ is defined as its limit for $j \rightarrow n$, that is equal to $\epsilon /(1+\epsilon)$.

Corollary to Theorem 1.

$$
P\left\{\sup _{a \leqq F^{\prime}(x)} \frac{F_{n}(x)-F(x)}{F(x)}<\epsilon\right\}=N^{\prime}(\epsilon, a, n)
$$

where $N^{\prime}(\epsilon, a, n)$ is given by the above expression for $N(\epsilon, b, n)$ after replacing $b$ by $1-a$ in it.

(3.2) follows immediately from Theorem 1 if we put $b=1-a$ and replace $1-F(x)$ by $F(x)$ and $1-F_{n}(x)$ by $F_{n}(x)$ in it. 
THEOREM 2.

$$
P\left\{\sup _{P_{n}(x) \leqq 0} \frac{F(x)-F_{n}(x)}{1-F_{n}(x)}<\epsilon\right\}=M(\epsilon, b, n)
$$

where

$$
\begin{aligned}
M(\epsilon, b, n) & =1-\sum_{j=0}^{k} L(\epsilon, j, n) \\
& =\sum_{j=k+1}^{n} L(\epsilon, j, n), \quad 0<\epsilon<1
\end{aligned}
$$

and where $k=[n b]$, and $L(\epsilon, j, n)$ is defined as

$$
L(\epsilon, j, n)=\left(\begin{array}{l}
n \\
j
\end{array}\right)\left[1-\frac{j}{n}-\epsilon\left(1-\frac{j}{n}\right)\right]^{n-j}\left[\frac{j}{n}+\epsilon\left(1-\frac{j}{n}\right)\right]^{j-1} \epsilon
$$

and for $j=n, L(\epsilon, j, n)$ is defined as its limit for $j \rightarrow n$, that is as equal to $\epsilon$.

REMARK on Theorem 2 . It is interesting to note here that Theorem 2 can also be written as

$$
\begin{aligned}
\left\{\sup _{F_{n}(x) \leqq b} \frac{F(x)-F_{n}(x)}{1-F(x)}<z\right\} & =1-\sum_{j=0}^{k} K(z, j, n) \\
& =\sum_{j=k+1}^{n} K(z, j, n)
\end{aligned}
$$

where $k=[n b], z=\epsilon /(1-\epsilon), 0<\epsilon<1$ and $K(\cdot, j, n)$ is as it was defined in Theorem 1 . Thus $N(\cdot, b, n)$ of Theorem 1 and $M(\cdot, b, n)$ of Theorem 2 actually have the same form and they differ only in the definition of their $k$ 's respectively. Comparing (3.1) and (3.4) we see that this difference in the definition of their respective $k$ 's is due to the replacement of the fixed interval: $F(x) \leqq b$ of Theorem 1 by the random interval: $F_{n}(x) \leqq b$ of (3.4) in their respective "sup" statements. We will actually see that the limiting forms of (3.1), (3.3) and (3.4) are the same.

Corollary to Theorem 2.

$$
P\left\{\sup _{a \leqq F_{n}(x)} \frac{F_{n}(x)-F(x)}{F_{n}(x)}<\epsilon\right\}=M^{\prime}(\epsilon, a, n)
$$

where $M^{\prime}(\epsilon, a, n)$ is given by the expression for $M(\epsilon, b, n)$ after replacing $b$ by $1-a$ in it. 
(3.5) follows from (3.3) exactly the same way as (3.2) does from (3.1).

Similarly, as a corollary to (3.4), we have

$$
\begin{aligned}
P\left\{\sup _{a \leq P_{n}(x)} \frac{F_{n}(x)-F(x)}{F(x)}<z\right\} & =1-\sum_{j=0}^{k} K(z, j, n) \\
& =\sum_{j=k+1}^{n} K(z, j, n),
\end{aligned}
$$

and this is just another way of writing (3.5), where $k=[n(1-a)]$, $z=\epsilon /(1-\epsilon), 0<\epsilon<1$, and the relation of (3.6) to (3.2) is the same as that of (3.4) to (3.1).

A REMARK ON THE LIMITING FORMS OF ABOVE THEOREMS. In his fundamental paper [5], A. Rényi obtained, among many others, the following result:

$$
\begin{aligned}
\lim _{n \rightarrow \infty} P\left\{n^{1 / 2} \sup _{a \leqq P(x)} \frac{F_{n}(x)-F(x)}{F(x)}<y\right\}=\Phi\left(y(a /(1-a))^{1 / 2}\right) \\
= \begin{cases}(a / \pi)^{1 / 2} \int_{0}^{y(a /(1-a))^{1 / 2}} \exp \left(-t^{2} / 2\right) d t, & y>0, \\
0, & y \leqq 0,\end{cases}
\end{aligned}
$$

that is the limiting form of (3.2) with $\epsilon=y(n)^{-1 / 2}$.

Evidently, the following statement is also true:

$$
\lim _{n \rightarrow \infty} P\left\{n^{1 / 2} \sup _{r(x) \leqq b} \frac{F(x)-F_{n}(x)}{1-F(x)}<y\right\}=\Phi\left(y((1-b) / b)^{1 / 2}\right.
$$

where $\Phi(\cdot)$ is as given in (3.7), and this is the limiting form of (3.1) with $\epsilon=y n^{-1 / 2}$.

Using the Glivenko-Cantelli theorem:

$$
P\left\{\lim _{n \rightarrow \infty}\left(\sup _{-\infty<x<+\infty}\left|F_{n}(x)-F(x)\right|\right)=0\right\}=1,
$$

it can be very easily shown that the fixed intervals: $a \leqq F(x)$ and $F(x) \leqq b$ of (3.7) and (3.8) can be replaced by the random intervals: $a \leqq F_{n}(x)$ and $F_{n}(x) \leqq b$ under their indicated "sup"'s respectively and the same limit theorems hold. This implies that the limiting forms of (3.6) and (3.4), with $z=y n^{-1 / 2}$, are also given by (3.7) and (3.8) respectively. But (3.6) is just another way of writing (3.5) and the same is true about (3.4) in relation to (3.3). Thus the limiting form of (3.5) is also given by (3.7) and that of (3.3) by (3.8) with $\epsilon=y n^{-1 / 2}$ in both of them. 
Proof of Theorem 1. Keeping with the notation of $\$ 2$ it is clear that the distribution of the random variable of (3.1) is identical with that of $\sup _{Y \leq b}\left\{Y-G_{n}(Y)\right\} /(1-Y)$ and saying that

$$
\sup _{Y \leqq b} \frac{Y-G_{n}(Y)}{1-Y}<\epsilon
$$

is equivalent to saying: $Y<G_{n}(Y) /(1+\epsilon)+\epsilon /(1+\epsilon)$ for all $Y \leqq b$. From the definition of $G_{n}(Y)$ it follows that $Y<\left\{G_{n}(Y)+\epsilon\right\} /(1+\epsilon)$ for all $Y \leqq b$ occurs if and only if the ordered random sample

$$
0<Y_{1}^{*}<Y_{2}^{*}<\cdots<Y_{n}^{*}<1
$$

falls into the region

$$
\begin{aligned}
& Y_{j-1}^{*}<Y_{j}^{*}<\left(\frac{j-1}{n}+\epsilon\right) /(1+\epsilon) \text { for } j=1, \cdots, k+1, \\
& Y_{j-1}^{*}<Y_{j}^{*}<1 \quad \text { for } j=k+2, \cdots, n
\end{aligned}
$$

where $Y_{0}^{*}=0$ and $k$ is the greatest integer so that

$$
\left(\frac{k}{n}+\epsilon\right) /(1+\epsilon) \leqq b,
$$

that is $k=[n\{(1+\epsilon) b-\epsilon\}]$ and thus, to make $k \geqq 0, b$ is such that $0<\epsilon /(1+\epsilon) \leqq b$.

The density function of (3.10) is given by

$$
n ! d Y_{1}^{*} d Y_{2}^{*} \cdots d Y_{n}^{*}
$$

and the probability that (3.10) falls into the region (3.11) is given by

$$
\begin{aligned}
n ! \int_{Y_{0}^{*}=0}^{e /(1+\epsilon)} \int_{Y_{1}^{*}}^{1 / n(1+\epsilon)+e /(1+\epsilon)} & \cdots \int_{Y_{k}^{*}}^{k / n(1+\epsilon)+\epsilon /(1+e)} \int_{Y_{k+1}^{*}}^{1} \cdots \\
& \int_{Y_{n-1}^{*}}^{1} d Y_{n}^{*} \cdots d Y_{k+2}^{*} d Y_{k+1}^{*} \cdots d Y_{2}^{*} d Y_{1}^{*}
\end{aligned}
$$

with $\epsilon>0$ and $k=[n\{(1+\epsilon) b-\epsilon\}]$. Comparing this integral to that of (2.2) we conclude that it is equal to

$$
\begin{array}{r}
1-\sum_{j=0}^{k}\left(\begin{array}{l}
n \\
j
\end{array}\right)\left(1-\frac{j}{n(1+\epsilon)}-\frac{\epsilon}{1+\epsilon}\right)^{n-j}\left(\frac{j}{n(1+\epsilon)}+\frac{\epsilon}{1+\epsilon}\right)^{j-1} \frac{\epsilon}{1+\epsilon} \\
\quad=1-\sum_{j=0}^{k} K(\epsilon, j, n), \quad \epsilon>0, \quad k=[n\{(1+\epsilon) b-\epsilon\}]
\end{array}
$$


on using notation introduced in (3.1). This result can be checked by direct evaluation of (3.14). In order to show that $1-\sum_{j=0}^{k} K(\epsilon, j, n)$ $=\sum_{j-k+1}^{n} K(\epsilon, j, n)$ we will have to verify that

$$
\sum_{j=0}^{n} K(\epsilon, j, n)=1 \text { for all integers } n>0 .
$$

We consider

$$
\begin{aligned}
& \sum_{j=0}^{n} K(\epsilon, j, n) \\
& =\{1 / n(1+\epsilon)\}^{n-1} \frac{\epsilon}{1+\epsilon} \sum_{j=0}^{n}\left(\begin{array}{c}
n \\
j
\end{array}\right)(n-j)^{n-j}(j+n \epsilon)^{j-1} \\
& =\left\{1 / n(1+\epsilon)^{n-1} \frac{\epsilon}{1+\epsilon} \sum_{i=-1}^{n-1}\left(\begin{array}{c}
n \\
i+1
\end{array}\right)(n-1-i)^{n-i-1}(n \epsilon+1+i)^{i}\right.
\end{aligned}
$$

where $i=j-1$ and continuing

$$
=\{1 / n(1+\epsilon)\}^{n-1} \frac{\epsilon}{1+\epsilon}\left[\frac{n^{n}}{n \epsilon}+\sum_{i=0}^{n-1}\left(\begin{array}{c}
n \\
i+1
\end{array}\right)(\alpha+i)^{i}(\beta-i)^{n-i-1}\right]
$$

where $\alpha=\epsilon n+1$ and $\beta=n-1$ and applying Lemma 3 we immediately get (3.16). This completes the proof of Theorem 1 .

Proof of Theorem 2. On the lines of proof of Theorem 1 we can say here that the event of (3.3) is equivalent to (3.10) falling into the region

$$
\begin{aligned}
& Y_{j-1}^{*}<Y_{j}^{*}<\frac{j-1}{n}+\epsilon\left(1-\frac{j-1}{n}\right) \text { for } j=1,2, \cdots, k+1, \\
& Y_{j-1}^{*}<Y_{j}^{*}<1 \quad \text { for } j=k+2, \cdots, n,
\end{aligned}
$$

where $Y_{0}^{*}=0$ and $k$ is greatest integer such that $k / n+\epsilon(1-k / n)$ $\leqq b+\epsilon(1-b)<1$ and thus $k=[n b]$. An integral expression of type (3.14) immediately gives, by comparison to (2.2), $M(\epsilon, b, n)$ of (3.3) as equal to $1-\sum_{j=0}^{k} L(\epsilon, j, n), 0<\epsilon<1$ and $k=[n b]$. That $M(\epsilon, b, n)$ of (3.3) is also equal to $\sum_{j=k+1}^{n} L(\epsilon, j, n)$ follows from

$$
\sum_{j=0}^{n} L(\epsilon, j, n)=1 \quad \text { for all integer } n>0,
$$

which can be verified by applying Lemma 3 again the way we did when proving (3.16), or it can be seen immediately from (3.4) and (3.16). This completes the proof of Theorem 2 . 
THEOREM 3.

$$
\begin{aligned}
P\left\{\sup _{F(x)<1} \frac{F(x)-F_{n}(x)}{1-F(x)}<\epsilon\right\} & =1-\sum_{j=0}^{n-1} K(\epsilon, j, n) \\
& =\frac{\epsilon}{1+\epsilon}, \quad \epsilon>0 .
\end{aligned}
$$

The first equality of (3.19) can be verified exactly the same way as Theorem 1 and the second equality follows from (3.16) and from the definition of $K(\epsilon, j, n)$ for $j=n$.

As a corollary to Theorem 3 we also have that

$$
\left\{\sup _{0<F(x)} \frac{F_{n}(x)-F(x)}{F(x)}<\epsilon\right\}=\frac{\epsilon}{1+\epsilon}, \quad \epsilon>0 .
$$

Theorem 3 and its corollary (3.20) are not new and were first proved by Daniels [4].

TheOREM 4.

$$
\begin{aligned}
P\left\{\sup _{P_{n}(x)<1} \frac{F(x)-F_{n}(x)}{1-F_{n}(x)}<\epsilon\right\} & =1-\sum_{j=0}^{n-1} L(\epsilon, j, n) \\
& =\epsilon, \quad 0<\epsilon<1 .
\end{aligned}
$$

The first equality of (3.21) is verified exactly the same way as Theorem 2 while the second one follows from (3.18) and definition of $L(\epsilon, j, n)$ for $j=n$.

Theorem 4 can also be written as

$$
P\left\{\sup _{P_{n}(x)<1} \frac{F(x)-F_{n}(x)}{1-F(x)}<z\right\}=\frac{z}{1+z},
$$

where $z=\epsilon /(1-\epsilon), 0<\epsilon<1$, and it is only proper that this form is equivalent to (3.19) for none of the results depend on $n$ and we have already remarked that the fixed interval: $F(x)<1$ can be replaced by the random one: $F_{n}(x)<1$ under respective "sup"'s in the limit as $n \rightarrow \infty$ and the same statements must hold. So in this case they cannot but be equal for finite $n$ too, both of the results being independent of $n$.

As corollaries to Theorem 4 we also have

$$
P\left\{\sup _{0<P_{n}(x)} \frac{F_{n}(x)-F(x)}{F_{n}(x)}<\epsilon\right\}=\epsilon, \quad 0<\epsilon<1
$$

and 


$$
P\left\{\sup _{0<F_{n}(x)} \frac{F_{n}(x)-F(x)}{F(x)}<z\right\}=\frac{z}{1+z},
$$

where $z=\epsilon /(1-\epsilon), 0<\epsilon<1$. (3.24) compares with (3.20) in the same sense as (3.22) does with (3.19).

Appendix. Papers [7], [8] and [9] in the list of references were brought to my attention by the referee of this paper. The problem treated in [8] and [9] is different from that of this paper, though closely related to it. To illustrate this point, in the terminology of this paper, [8] and [9] examine the exact distribution of the random variable

$$
\sup _{0<F(x) \leqq a} \frac{F_{n}(x)-F(x)}{F(x)}
$$

while I deal with

$$
\sup _{0<a \leqq F(x)} \frac{F_{n}(x)-F(x)}{F(x)}
$$

in (3.2). The method of proof is also not the same.

There is an overlap between [7] and this paper. Theorem 1 of both papers deals with the exact distribution of the same random variable. However, their respective conclusions appear to be somewhat different. I believe the normalizing factor

$$
\left[\sum_{m=k}^{n}\left(\begin{array}{l}
n \\
m
\end{array}\right) b^{m}(1-b)^{n-m}\right]^{-1}
$$

is missing from formula (11) of Ishii's paper [7] and with this correction his result is correct. The same is true, mutatis mutandis, regarding his formula (16).

In this light it seems that (3.1) of this paper is a simpler version of Ishii's corrected formula (11).

\section{REFERENCES}

1. N. H. Abel, Oevres completes, Christiania, C. Groendahl, 1839.

2. Z. W. Birnbaum and Ronald Pyke, On some distributions related to the statistic $D_{n}^{+}$, Ann. Math. Statist. 29 (1958), 179-187.

3. Z. W. Birnbaum and Fred H. Tingey, One-sided confidence contours for probability distribution functions, Ann. Math. Statist. 22 (1951), 592-596.

4. H. E. Daniels, The statistical theory of the strength of bundles of threads. I, Proc. Roy. Soc. Ser. A 183 (1945), 405-435.

5. Alfred Renyi, On the theory of order statistics, Acta Math. Acad. Sci. Hungar. 4 (1953), 191-231. 
6. N. Smirnov, Sur les ecarts de la courbe de distribution empirique, Mat. Sb. (N.S.) 6 (48) (1939), 3-26.

7. Goro Ishii, On the exact probabilities of Renyi's tests, Ann. Inst. Statist. Math. Tokyo 11 (1959), 17-24.

8. Li-Chien Chang, On the ratio of an empirical distribution function to the theoretical distribution function, Acta Math. Sinica 5 (1955), 347-368; English transl., Selected Transl. Math. Statist. and Prob., Vol. 4, pp. 17-38, Inst. Math. Statist. and Amer. Math. Soc., Providence, R. I., 1963.

9. S. C. Tang, Some theorems on the ratio of empirical distribution to the theoretical distribution, Pacific J. Math. 12 (1962), 1107-1114.

Princeton University

\section{BANACH ALGEBRAS OF SCALAR-TYPE ELEMENTS}

\section{BERTRAM WALSH}

In [5] H. Schaefer asked whether a locally convex algebra having the property that each of its elements is "spectral" (i.e. can be represented as the integral of some measurable function with respect to some spectral measure [is of "scalar type" in the sense of Dunford]) must necessarily be a commutative algebra. This note answers the question only for Banach algebras, but shows that the answer is affirmative under a hypothesis less restrictive than that which Schaefer suggests, and also (via a theorem of Katznelson [3]) that in fact a Banach algebra satisfying this hypothesis is automatically isomorphic to $\mathrm{e}(M), M$ its maximal ideal space. The result may also be viewed then as a variant of Katznelson's, in which commutativity and semisimplicity are not required $a$ priori.

For simplicity's sake the presence of an identity is assumed in all algebras discussed below, and homomorphisms are assumed to carry identities to identities. The modifications necessary to dispense with these assumptions are straightforward.

Let $\mathfrak{A}$ be a real or a complex Banach algebra. We shall say that an element $a \in \mathfrak{A}$ is prescalar under the following circumstances:

(1) If $\mathfrak{A}$ is a real Banach algebra, we require that the spectrum of $a$ (as defined for real Banach algebras via complexification-see [4, p. 28]) be real, and that there be a homomorphism $h_{a}: \mathfrak{C}_{R}(\sigma(a)) \rightarrow \mathfrak{A}$ (where as usual $\mathfrak{C}_{R}(\sigma(a))$ is the sup-norm algebra of continuous real-

Received by the editors November 2, 1964. 